\title{
ISO OBSERVATIONS OF CIRCUMSTELLAR MATERIAL
}

\author{
H.J. HABING \\ Sterrewacht \\ Huygens Laboratorium \\ Postbus 9513 \\ 2300 RA Leiden
}

\section{Introduction}

Circumstellar matter is found almost exclusively around young stars that have not yet reached the main sequence and around old stars that have left this sequence. Some main sequence stars still carry the remnants of a disk of dustlike material as a remnant of their time of formation.

\section{Circumstellar matter around young and middle-aged stars}

\subsection{YOUNG STARS: TTAU AND HAEBE STARS}

Using ISOPHOT Ábrahám et al. (1997) have detected emission from three out of seven observed Herbig $\mathrm{AeBe}(\equiv \mathrm{HAeBe}$ ) stars. The emission is stronger than the photosphere can emit and this suggests that the presence of dusty disks in HAeBe and TTau stars is a common property. Waelkens (1997) suggested that HAeBe stars are the progenitors of $\beta$ Pic stars. He attributes certain spectral features in the young star HD 100546 and in comet Hale-Bop to crystalline silicates; the mechanism that induced the crystalisation of the silicates is unknown.

\subsection{THE VEGA PHENOMENON: DUST DISKS AROUND MAIN-SEQUENCE STARS}

Heinrichsen et al (1997) confirm IRAS and ground based (inc. KAO) measurements of the dust disk around Vega. They add a $170 \mu \mathrm{m}$ measurement and determine sizes at $60 \mu \mathrm{m}$ (a diameter of $22 \pm 3^{\prime \prime}$ or $\left.86 \mathrm{AU}\right)$ and at $90 \mu \mathrm{m}\left(36 \pm 3^{\prime \prime}\right.$ or $\left.140 \mathrm{AU}\right)$, respectively.

Habing et al. (1996) and Dominik (1997) report first results of an ISOPHOT programme to establish the fraction of main-sequence stars with a Vega-like excess. So far the indications are that about $1 / 3$ of their candidates show excess emission, $1 / 3$ does not and $1 / 3$ of their cases are undecided. The star $\rho^{1} \mathrm{Cnc}$, that is known to have a planet also shows a $60 \mu \mathrm{m}$ excess emission: apparently the presence of a planet does not exclude the presence of a dust disk.

\section{Circumstellar envelopes around evolved stars of low and intermediate mass}

Calculations of stellar interiors and a large body of observational data (Habing, 1996) show that stars with a mass below about $6 M_{\odot}$ will ultimately, and for a short time only (10 $\mathrm{yr}$ ) become a pulsating red variable and eject matter at a low speed, at first at a low but later at a very high rate. ISO's contributions are flux density measurements at long wavelengths and spectroscopy through the full range from 2 tot $200 \mu \mathrm{m}$.

\subsection{AGB STARS WITH MODEST MASS LOSS RATES}

Visible Long-Period Variables with periods over 300 days have circumstellar envelopes of low optical depth: the star itself is easily observed. Heske et al. carry out a low-resolution spectral survey of RGB and AGB stars in the hope of finding distinctive properties of the various categories of red giants: a perhaps not glamorous but very important study. 
Here in Japan Tsuji and co-workers have been leading world experts in the study of very cool stars. Discussing results obtained with ITRS, Tsuji emphasized once again the existence of a cool layer on top of the photosphere and the large effect this layer has on the infrared spectrum. High-quality spectra between 2 and 20 micron show convincingly that late M- and C-giants have considerable amounts of gas at a distance of a few stellar radii from the photosphere and with a temperature of several hundred $\mathrm{K}$.

There are still large problems in the construction of very extended and dynamical model atmospheres for variable red giants; fresh attempts are being made by a group in Vienna (Hron et al. 1997).

\subsection{AGB STARS WITH HIGH MASS LOSS RATES}

SWS spectra of stars with all kinds of mass-loss rates are being studied by various consortia of astronomers in the Netherlands. The spectra obtained with ISO are very rich. The extraction of astrophysical information is beginning; they should lead to a much better insight in the circumstellar environment.

\subsection{POST-AGB STARS AND PLANETARY NEBULAE}

I skip the discussion of Post-AGB stars and of planetary nebulae but I cannot escape the remark that ISO continues to make interesting, and also beautiful, measurements. See for example the poster by Pierre Cox et al. in the events hall. In the coming years we may expect great leaps forward in understanding both Post-AGB stars and Planetary Nebulae.

\section{Evolved stars of high mass}

In their last years high-mass stars also produce much circumstellar material. One group of rare and interesting objects are the Luminous Blue Variables, LBVs: stars like $\eta$ Carinae, AG Carinae and $R$ Doradus. New members of this group are now discovered via their circumstellar envelope. IRAS data prove to be useful, but the envelope of at least one LBV has been found at radio wavelengths (Wendker et al., 1977).

A special case that must be mentioned is the star IRC +10420 , a supergiant with a strong 1612 $\mathrm{MHz} \mathrm{OH}-m$ maser. On theoretical grounds Elitzur, Goldreich and Scoville proposed in 1976 that the maser is pumped radiatively and state that the most important $\mathrm{OH}$ absorption line of this pump is at $34.6 \mu \mathrm{m}$. Observations with the SWS and LWS, reported by Sylvester et al. (1997) now confirm the theoretical predictions for the first time.

\section{References}

Ábrahám, P., Leinert, Ch., Lemke, D., Burkert, A., Henning, Th. 1997, in "ISO's View of Stellar Evolution", eds. L. Waters and $\mathrm{C}$. Waelkens (in print)

Dominik, C. 1997, in "ISO's View of Stellar Evolution", eds. L. Waters and C. Waelkens (in print)

Elitzur, M., Goldreich, P., Scoville, N. 1976, ApJ 205, 384

Habing, H.J. 1996, The Astron. Astrophys. Rev. 7, 97

Habing, H.J., Bouchet, P., Dominik, C. et al., 1996, A\&A 315, L233

Heinrichsen, I., Walker, H.J. 1997, in "ISO's View of Stellar Evolution", eds. L. Waters and C. Waelkens (in print)

Hron, J., Loidl, R., Jorgensen, U.G., Kerschbaum, F. 1977, in "ISO's View of Stellar Evolution", eds. L. Waters and C. Waelkens (in print)

Sylvester, R.J., Barlow, M.J., Nguyen-Q-Rieu, Liu, X.-W., Skinner, C.J., Cohen, R.J., Lim, T., Cox, P., TruongBach, Smith, H.A., Habirng, H.J. 1997, MNRAS, in print

Walker, H.J., Heinrichsen, I., Richards, P.J. 1997, in "ISO's View of Stellar Evolution", eds. L. Waters and C. Waelkens (in print)

Wendker, H., Heske, A. et al, in "ISO's View of Stehlar Evolution", eds. L. Waters and C. Waelkens (in print) 